\title{
Gagnants des résumés du Congrès 2012 des étudiants de la Société canadienne d'épidémiologie et de biostatistique
}

\section{Préface}

Les responsables de la publication Maladies chroniques et blessures au Canada (MCBC) ont été heureux d'organiser, une fois de plus, le concours des résumés pour étudiants dans le cadre du Congrès des étudiants de la Société canadienne d'épidémiologie et de biostatistique (SCEB), qui a eu lieu à l'Université de la Saskatchewan, en mai 2012. Un comité éditorial de l'Agence de la santé publique du Canada a jugé 42 présentations de résumé et choisi les 7 meilleurs, qui sont publiés dans ce numéro.

Le comité éditorial était formé des membres suivants :

- Howard Morrison, Ph.D., rédacteur en chef, MCBC

- Kenneth Johnson, Ph. D., épidémiologiste principal

- Ania Syrowatka, M. Sc., épidémiologiste

- Michelle Tracy, M.A., gestionnaire de la rédaction, $\mathrm{MCBC}$

Les résumés choisis ont été jugés en fonction de leur originalité, de leur clarté, de leur excellence scientifique et technique et des incidences potentielles. Les questions suivantes ont servi de balises aux juges :

1. Est-ce pertinent pour les maladies chroniques ou les blessures?

2. Les données sont-elles canadiennes? Si ce n'est pas le cas, les auteurs replacent-ils la question dans le contexte canadien?

3. L'étude est-elle pertinente à l'échelle nationale? Les études locales ne suscitent de l'intérêt que si elles fournissent suffisamment de détails pour être utiles aux chercheurs de l'extérieur.

4. L'étude répond-elle à une question de santé publique?
5. L'étude est-elle rigoureuse du point de vue scientifique?

6. S'agit-il de méthodes nouvelles ou de résultats nouveaux, ou est-ce une amélioration importante par rapport aux études précédentes sur la même question?

7. Pouvez-vous imaginer d'autres circonstances raisonnables dans le cadre desquelles un auteur différent ferait référence à cette étude?

Depuis 2009, MCBC a collaboré avec la SCEB pour parrainer des occasions de publication pour les étudiants. Les responsables de MCBC sont fiers de collaborer avec la SCEB encore cette année et d'encourager les étudiants dans leurs efforts de publication. Au nom de l'équipe éditorial de MCBC, j'aimerais remercier tous les étudiants qui ont soumis leurs résumés et féliciter les gagnants. La publication d'un résumé dans une revue à comité de lecture constitue un bon départ dans la publication scientifique! Nous espérons recevoir dans l'avenir des articles de recherche complets.

Michelle Tracy, M.A.

Gestionnaire de la rédaction, Maladies

chroniques et blessures au Canada 


\title{
L'effet du genre et du milieu de vie sur l'autoévaluation de la santé mentale des chefs de famille monoparentale
}

\author{
A. Banerjee, M.B.B.S. (1); B. Janzen, Ph. D. (1)
}

Introduction : Les chefs de famille monoparentale, l'un des groupes les plus vulnérables sur le plan socio-économique au Canada, rapportent de façon constante un état de santé mentale moins satisfaisant que les parents vivant ensemble. La plupart des études évaluant leur santé mentale ne prennent pourtant pas en considération leur milieu de vie, ou se concentrent exclusivement sur ceux vivant en milieu urbain, malgré le nombre croissant de données indiquant que le milieu de vie (le degré de ruralité) est un déterminant de l'état de santé et alors que, selon les données de 2006, le pourcentage de chefs de famille monoparentale en milieu rural au Canada dépassait légèrement les $13 \%$. De plus, on connaît peu l'état de santé mentale des pères monoparentaux, même si leur nombre ne cesse d'augmenter au Canada.

Objectif : Déterminer si l'état de santé mentale des chefs de famille monoparentale varie selon le genre ou la ruralité, et quels sont les facteurs (p. ex., économiques, sociaux) contribuant à ces variations de la santé mentale selon le sexe ou la ruralité.

Méthodologie : Pour cette analyse, nous avons sélectionné parmi les participants à l'Enquête sur la santé dans les collectivités canadiennes de 2007-2008 (fichier maître) un sous-échantillon de chefs de famille monoparentale âgés de 18 à 64 ans ayant au moins un enfant de moins de 25 ans vivant avec eux. La variable dépendante était l'autoévaluation de l'état de santé mentale, et les principales variables indépendantes étaient le sexe et le lieu de résidence, cette dernière variable étant fondée sur la classification en zones d'influence des régions métropolitaines (ZIM) de Statistique Canada. Les covariables étaient l'âge, le statut d'emploi, le revenu familial, l'accession à la propriété, la sécurité alimentaire et le sentiment d'appartenance à la collectivité. Une série d'analyses de régression logistique unidimensionnelle, bidimensionnelle et multidimensionnelle a été effectuée en vue de répondre aux questions de recherche. La pondération de l'échantillonnage et un programme bootstrap d'estimation de la variance ont été utilisés pour gérer la stratégie complexe d'échantillonnage.

Résultats : L'échantillonnage (pondéré) était constitué de 1024856 chefs de famille monoparentale. Les mères monoparentales constituaient $81 \%$ de l'échantillon, et la majorité des chefs de famille monoparentale (86 \%) vivaient en milieu urbain. Dans l'ensemble, 9,3 \% des mères monoparentales et 7,0 \% des pères monoparentaux ont qualifié leur état de santé mentale comme étant passable ou médiocre. Le pourcentage de pères monoparentaux ayant un état de santé mentale passable ou médiocre était de 6,7 \% dans les régions métropolitaines de recensement et agglomérations de recensement (RMR et AR), de 11,0\% dans les zones d'influence des régions urbaines (ZIM) forte ou modérée et de 4,6 \% dans les ZIM faible ou nulle. Les pourcentages de mères monoparentales ayant un état santé mentale passable ou médiocre étaient de 9,5\% (RMR et AR), de 7,9\% (ZIM forte ou modérée) et de 8,2\% (ZIM faible ou nulle). L'étude des résultats préliminaires suggère des variations dans l'état de santé mentale autoévalué et dans l'accès à des ressources économiques et sociales (emploi, sécurité alimentaire, sentiment d'appartenance, etc.) selon le genre et le degré de ruralité. D'autres analyses utilisant des techniques appropriées d'estimation de la variance demeurent nécessaires pour déterminer si ces différences sont statistiquement significatives. De plus, il faudrait effectuer une analyse de régression logistique multiple pour déterminer si les différences observées dans l'état de santé mentale des chefs de famille monoparentale liées au genre ou à la résidence, ou aux deux, demeurent statistiquement significatives après un ajustement pour les principales covariables.

Conclusion : Les résultats de cette étude enrichissent les connaissances sur les expériences des chefs de famille monoparentale au Canada et pourront servir à l'élaboration de politiques mieux ciblées en vue d'améliorer la santé mentale des chefs de famille monoparentale.

Mots-clés : politique en matière de santé, politique sociale, santé mentale, épidémiologie sociale, épidémiologie comportementale, Enquête sur la santé dans les collectivités canadiennes

Rattachement des auteurs :

1. Département de santé communautaire et d'épidémiologie, Collège de médecine, Université de la Saskatchewan (Saskatchewan), Canada

Correspondance : Ankona Banerjee; courriel : asb426@mail.usask.ca 


\title{
Lien entre les activités sédentaires et l'idéation suicidaire chez les adolescents et les jeunes adultes au Canada
}

\author{
M.-A. Bélair, B. Sc. S. (2); I. Colman, Ph.D. (2)
}

Introduction : Le suicide est la deuxième cause de décès en importance chez les 15 à 24 ans au Canada. Le taux de suicide demeure constant parmi les adolescents canadiens (21,4\%) malgré des taux décroissants dans d'autres pays développés. Plus de $50 \%$ des adolescents qui se suicident souffrent d'un trouble dépressif majeur.

Objectif : Déterminer s'il existe un lien entre l'activité sédentaire et l'idéation suicidaire chez les adolescents et les jeunes adultes de 15 à 24 ans.

Méthodologie : Nous avons sélectionné pour cette analyse 7914 adolescents et jeunes adultes de 15 à 24 ans à partir d'un échantillon initial de 8356 sujets ayant participé à l'Enquête sur la santé dans les collectivités canadiennes (ESCC) - Cycle 4 (2007-2008). Nous avons effectué des tests de Breslow-Day pour la modification de l'effet en vue de déterminer la nécessité d'effectuer une stratification et une analyse de régression logistique multidimensionnelle dans l'évaluation du lien entre activité sédentaire et idéation suicidaire à vie. L’activité sédentaire a été classée en trois catégories en fonction de sa durée hebdomadaire : 0 à 15,15 à 34 et 35 heures et plus par semaine.
Résultats : Les sujets sédentaires durant 15 à $34 \mathrm{~h}$ par semaine avaient un rapport de cote (RC) d'idéation suicidaire à vie 1,18 fois plus élevé (intervalle de confiance [IC] à $95 \%$ : 0,99 à 1,41) que celui des sujets sédentaires entre 0 et 15 h par semaine, alors que les sujets sédentaires durant 35 h et plus par semaine avaient un RC 1,41 fois plus élevé (IC à $95 \%: 1,15$ à 1,74). Après ajustements pour le sexe, l'âge, l'autoperception de l'état de santé, l'autoperception de l'état de santé mentale et l'indice de masse corporelle (IMC), et après la modélisation de l'interaction entre le sexe et l'autoperception de l'état de santé et entre le sexe et l'IMC, le lien entre l'idéation suicidaire et l'activité sédentaire des adolescents et des jeunes adultes de la catégorie $35 \mathrm{~h}$ et plus par semaine est demeuré significatif avec un RC ajusté de 1,33 (IC à $95 \%$ : 1,06 à 1,68), alors que pour les sujets de la catégorie 15 à $34 \mathrm{~h}$ par semaine, le lien était non significatif avec un RC ajusté de 1,11 (IC à $95 \%$ : 0,92 à 1,35). Pour interpréter les termes de l'interaction, nous avons utilisé des modèles stratifiés en fonction du sexe. Chez les jeunes hommes percevant leur état de santé comme étant médiocre ou passable, le RC d'idéation suicidaire à vie était de 1,26 (IC à $95 \%$ : 0,82 à 1,26), alors que chez les jeunes femmes il était de 2,33 (IC à $95 \%$ : 1,68 à 3,23) par rapport au modèle de référence (autoperception d'un état de santé bon, très bon ou excellent). Une augmentation de 10 unités d'IMC a entraîné une réduction de la cote d'idéation suicidaire à vie de 0,97 (IC à $95 \%$ : 0,73 à 1,28 ) chez les jeunes hommes, alors qu'elle a entraîné une augmentation de la cote d'idéation suicidaire à vie de 1,58 (IC à $95 \%: 1,29$ à 1,92$)$ chez les jeunes femmes.

Conclusion : Un lien existe entre les degrés d'activité sédentaire et l'idéation suicidaire à vie chez les adolescents et les jeunes adultes ayant une activité sédentaire pendant 35 heures et plus par semaine. Cette observation est préoccupante puisqu'un pourcentage de plus en plus élevé d'adolescents et de jeunes adultes consacrent plus davantage de temps à une activité sédentaire. Cependant, en raison de la nature transversale des données de l'ESCC, nous ne pouvons pas nous prononcer sur la direction de ce lien. Il faudrait une recherche plus poussée à l'aide de données longitudinales.

Mots-clés : santé mentale, Enquête sur la santé dans les collectivités canadiennes, dépression, suicide 


\title{
État de santé et utilisation des services de soins de santé chez les personnes itinérantes atteintes d'une maladie mentale : concordance entre déclarations et dossiers médicaux administratifs dans le cadre du projet multicentrique At Home/Chez Soi
}

\author{
A. Hinds, M. Sc. (3); J. Distasio, Ph. D. (4); P. J. Martens, Ph. D. (5); M. Smith, M. Sc. (5)
}

Introduction : Les personnes itinérantes dont l'état de santé est précaire utilisent souvent les services de soins de santé.

Objectif : Étudier l'état de santé et l'utilisation des services de soins de santé et la fourniture de médicaments sur ordonnance des personnes itinérantes atteintes de maladie mentale et comparer leurs déclarations aux données administratives relatives aux demandes de remboursement, en vue d'estimer le degré de concordance entre les deux sources de données.

Méthodologie : Nous avons comparé les données de référence d'une enquête réalisée auprès de 100 participants de la section de Winnipeg du projet At Home/Chez soi de la Commission de la santé mentale du Canada aux dossiers médicaux administratifs sans identifiants conservés au service d'archives du Centre d'élaboration de la politique des soins de santé du Manitoba. Nous avons analysé les caractéristiques des participants, leurs antécédents d'itinérance et leur utilisation des services de soins de santé, ainsi que leur état de santé relativement à l'asthme, à l'hypertension, à l'arthrite et au diabète (en utilisant des définitions préalablement validées). Les participants ont été classés de manière similaire sur la base de leurs réponses à l'enquête. Le degré de concordance entre les deux ensembles de données a été évalué à l'aide d'une tabulation en croix et du coefficient kappa $[\kappa]$.

Résultats : Nous avons pu coupler $100 \%$ des réponses fournies par les personnes itinérantes aux données archivées. En une année, $97 \%$ des participants avaient consulté au moins une fois un médecin dans un service de soins ambulatoires, le taux ajusté pour le sexe et l'âge étant de 14,82 par année-personne (taux global au Manitoba : 4,99 par année-personne); $34 \%$ avaient été hospitalisés (taux ajusté de congé d'hôpital : 491 par mille annéespersonnes par rapport au taux global au Manitoba de 137 par mille années-personnes) et $95 \%$ avaient reçu un médicament sur ordonnance au moins une fois, $65 \%$ étant pour un médicament traitant une maladie du système nerveux (la majorité étant des psycholeptiques). Le degré de concordance entre les sources de données concernant les maladies a varié de faible $(\kappa=0,27)$ pour l'arthrite à modéré $(\kappa=0,57)$ pour l'hypertension. Les sujets étaient plus susceptibles d'être considérés comme étant atteints de l'une des quatre maladies sélectionnées si on se fiait aux données administratives plutôt qu'aux données de l'enquête.

Conclusion : L'utilisation des services de soins de santé et de médicaments fournis sur ordonnance par les personnes itinérantes ayant participé à l'enquête était élevée comparativement à la population générale. Le degré de concordance entre les deux sources de données variait de faible à modéré pour la détection de maladies. Les chercheurs évaluant des personnes itinérantes atteintes d'une maladie mentale devraient envisager d'utiliser des sources de données variées pour estimer la prévalence d'une maladie et l'utilisation des services de soins de santé.

Mots-clés : santé mentale, méthodes épidémiologiques, utilisation des services de soins de santé, dossiers médicaux administratifs, itinérance 


\title{
Modélisation des coûts des épisodes de soins dans les cas d'exacerbation de la maladie pulmonaire obstructive chronique
}

\author{
J. P. Kuwornu, M. Sc. (6); L. M. Lix, Ph. D. (6, 7); J. M. Quail, Ph. D. (6, 7); E. Wang, M. Sc. (7); M. Osman, M.A. (7)
}

Introduction : Un épisode de soins (EdS) est un ensemble de services de soins de santé associés à une maladie aiguë ou chronique. Les EdS sont utilisés pour évaluer les variations de coûts et d'utilisation de différentes stratégies thérapeutiques. Les modèles prédictifs de l'utilisation et des coûts peuvent être utilisés pour déterminer quelles stratégies thérapeutiques donneront les résultats optimaux. Cependant, une difficulté importante dans l'élaboration de prédictions valides et précises est le choix d'un modèle statistique approprié.

Objectif : Comparer différents modèles statistiques pour prédire les coûts des EdS dans les cas d'exacerbation de la maladie pulmonaire obstructive chronique (MPOC).

Méthodologie : Les données de l'étude incluaient les congés de l'hôpital, les demandes de remboursement des médecins, les dossiers relatifs aux médicaments fournis sur ordonnance et les registres de population de la Saskatchewan. La cohorte de l'étude était constituée de personnes de 35 ans et plus ayant reçu un diagnostic de MPOC selon les demandes de remboursement des hôpitaux ou des médecins. Nous avons repéré les EdS amorcés par une hospitalisation pour un diagnostic principal de MPOC de 2000-2001 à 2009-2010, et calculé les coûts totaux pour l'hospitalisation, les médecins et les médicaments pour chaque EdS, avec ajustement en fonction de l'inflation. Nous avons comparé le modèle marginal pour l'équation d'estimation généralisée (EEG) et des modèles à effets aléatoires à une distribution binomiale gamma ou négative pour le coût moyen d'EdS et au modèle de régression quantile pour les coûts médians d'EdS. Les covariables incluaient les variables personnelles, socioéconomiques et pathologiques.

Résultats : Nous avons repéré dans la cohorte de l'étude (n = 41 848) 20999 EdS amorcés par une hospitalisation en raison d'une exacerbation de MPOC. L'âge moyen des sujets atteints de MPOC était de 71 ans (écart-type [É.-T.] : 12) et le pourcentage de sujets de sexe masculin était de $53 \%$. La courbe des coûts totaux était très asymétrique. Le coût médian total était de 4506 \$, alors que le coût moyen était de 7968 \$ (É.-T. : 13354 \$). La valeur médiane était plus élevée lors des épisodes ( $n=2400)$ au cours desquels le patient est décédé (8 380 \$) qu'au cours des épisodes où le patient a survécu ( $n=18$ 599; 4400 \$). Le modèle à effets aléatoires et le modèle EEG ne correspondaient pas à la distribution gamma. Le modèle de distribution binomiale négative correspondait bien aux données de la somme des carrés des écarts. Toutes les covariables de ce modèle étaient statistiquement significatives, à l'exception du sexe $(p=0,8179)$ et de l'âge ( $p=0,0610)$. Le modèle de régression quantile correspondait également; seul le score de comorbidité de Charlson n'était pas statistiquement significatif $(p=0,5791)$.

Conclusion : Le modèle de régression quantile et le modèle marginal avec une distribution binomiale négative semblent être des méthodes valides étant donné le faible pourcentage de sujets associés à des coûts d'EdS élevés en raison d'une MPOC. Les modèles ont donné divers résultats relativement à l'importance des covariables. Le choix de modèles influence les caractéristiques des patients associées aux coûts des soins de santé et aux démarches thérapeutiques, et peut mener à différentes conclusions concernant les stratégies thérapeutiques optimales chez les patients atteints de MPOC.

Mots-clés : analyse longitudinale, méthodes épidémiologiques, épidémiologie des maladies respiratoires

Rattachement des auteurs :

6. École de santé publique, Université de la Saskatchewan, Saskatoon (Saskatchewan), Canada

7. Conseil sur la qualité des soins de santé, Saskatoon (Saskatchewan), Canada

Correspondance : John Paul Kuwornu; courriel : paul.kuwornu@usask.ca 


\title{
Caractéristiques associées à une douleur accrue et à une récupération fonctionnelle réduite trois à cinq ans après une arthroplastie totale du genou
}

\author{
J. E. Mollins, M. Sc. (8); C. A. Jones, Ph. D. (9, 10); M. Clark, M.D. (11); L. Beaupré, Ph. D. $(9,10)$
}

Introduction : La fréquence des arthroplasties totales du genou (ATG) pratiquées au Canada s'accroît de façon constante; pourtant, de $9 \%$ à $19 \%$ des patients ayant subi une ATG bénéficient d'une amélioration légère ou nulle quant au fonctionnement physique et à l'atténuation de la douleur après l'intervention. Il n'existe pas de consensus concernant les facteurs associés à ces résultats insatisfaisants. La détermination des caractéristiques des patients associées à une douleur accrue et à un état fonctionnel négatif après l'intervention pourrait aider à cibler les patients moins susceptibles de bénéficier de cette intervention chirurgicale. Si ces facteurs sont modifiables, ils pourraient être corrigés avant l'ATG en vue d'améliorer les résultats postopératoires. S'ils ne sont pas modifiables, les patients purraient être informés pour que leurs attentes postopératoires soient réalistes.

Objectif : Déterminer les caractéristiques des patients, modifiables ou non, susceptibles d'être associées à de faibles indices WOMAC (Western Ontario McMaster Osteoarthritis Index) relativement à la douleur et à la fonction physique, trois à cinq ans après une ATG.

Méthodologie : Il s'agit d'une analyse secondaire des données de l'Alberta Arthroplasty Study, un vaste essai clinique randomisé. Nous avons effectué des analyses descriptives de base et comparé les indices initiaux des patients qui répondaient au traitement et de ceux qui n'y répondaient pas, ainsi qu'une analyse de régression linéaire univariée pour les variables indépendantes : âge, sexe, distribu- tion dans les groupes, indice de masse corporelle (IMC), catégories de comorbidités ( 2 maladies et moins et 3 maladies et plus), présence de dorsalgie, état relatif au diabète, présence d'une pneumopathie, tabagisme, résultats initiaux au questionnaire SF-36 (Medical Outcomes Study 36item Short Form) sur la santé mentale, indices initiaux WOMAC relatifs au fonctionnement physique et indices initiaux WOMAC relatifs à la douleur. Cette étape initiale d'élaboration de modèle a été effectuée deux fois : une fois avec les indices WOMAC relatifs à la douleur et une fois avec les indices WOMAC relatifs à l'état fonctionnel comme variable dépendante. Une analyse de régression multivariée a ensuite été élaborée à l'aide de méthodes de sélection intentionnelle. La stabilité finale du modèle a été évaluée à l'aide de programmes de régression multiple ascendante et de régression multiple descendante pour déterminer la concordance entre les variables significatives. Les facteurs d'augmentation de la variance ont été calculés pour tester la colinéarité.

Résultats : En tout, 388 patients ont accepté de poursuivre l'évaluation de 3 à 5 années après l'ATG. Nous avons observé des améliorations significatives des indices WOMAC relatifs à la douleur et à l'état fonctionnel. Dans les analyses multivariées, un âge plus avancé, la présence de dorsalgie et l'embonpoint ou l'obésité étaient des indices d'un degré plus élevé de douleur et d'un état fonctionnel moindre. De meilleurs indices WOMAC relatifs à la douleur et de meilleurs indices SF-36 relatifs à la santé mentale avant l'intervention ont été associés à de meilleurs résultats quant à la douleur après l'ATG. De meilleurs indices WOMAC relatifs à l'état fonctionnel et de meilleurs SH-36 relatifs à la santé mentale avant l'intervention étaient prédictifs de meilleurs résultats fonctionnels après l'ATG. Les coefficients de détermination $\left(R^{2}\right)$ étaient de 0,15 pour le modèle de la douleur et de 0,19 pour le modèle de l'état fonctionnel.

Conclusion : Un âge plus avancé était associé à de moins bons résultats relativement à la douleur plusieurs années après l'ATG; cependant, des patients âgés ont présenté des améliorations quant à la douleur similaires à celles observées chez de jeunes sujets. Par conséquent, un âge avancé ne devrait pas être un facteur limitatif lorsqu'on évalue des candidats à une ATG. Un IMC plus élevé était aussi un facteur prédictif important de la douleur et de l'état fonctionnel à long terme. Les interventions pour prendre en charge l'IMC et la dorsalgie devraient être envisagées avant l'intervention afin d'améliorer au maximum les résultats de l'ATG. On devrait pouvoir réviser les attentes des patients concernant les résultats de l'ATG en se fondant sur ces facteurs de risque ciblés. Les faibles valeurs $R^{2}$ indiquent une capacité limitée du modèle à prédire les résultats 3 à 5 ans après l'intervention chirurgicale. Une recherche plus poussée pourrait inclure d'autres variables psychosociales dans les modèles fondés sur les interventions médicales lors de l'évaluation des résultats de l'ATG.

Mots-clés : épidémiologie clinique, analyse longitudinale, vieillissement

Rattachement des auteurs :

8. Faculté de la médecine de réadaptation, Université de l'Alberta, Edmonton (Alberta), Canada

9. Département de physiothérapie, Université de l'Alberta, Edmonton (Alberta), Canada

10. Alberta Innovates - Health Solutions, Edmonton (Alberta), Canada

11. Département de chirurgie, Université de l'Alberta, Edmonton (Alberta), Canada

Correspondance : Juliana Mollins; courriel : mollins@ualberta.ca 


\title{
Mesures de l'importance relative des variables pour les données non normales : application aux résultats déclarés par les patients à propos de leur qualité de vie liée à la santé
}

\author{
T. T. Sajobi, Ph. D. (12); B. M. Dansu, Ph. D. (12); L. M. Lix, Ph. D. (12)
}

Introduction : Les mesures de la qualité de vie liée à la santé (QdVLS) sont largement utilisées dans les essais cliniques pour évaluer l'efficacité de nouveaux traitements sur les plans physique, psychologique et social. Des mesures de l'importance des variables dérivées de l'analyse discriminante descriptive (ADD) et des méthodes d'analyse de la variance de plusieurs variables (MANOVA) ont été élaborées en vue d'évaluer l'importance du domaine pour les données de la QdVLS recueillies à un moment donné. Elles incluent les coefficients standardisés de la fonction discriminante, les coefficients du ratio discriminant et les $\mathrm{F}$ d'exclusion [F-to-remove]. Cependant, ces mesures peuvent donner un classement par ordre de grandeur incohérent des données sur la QdVLS caractérisées par des distributions non normales.

Objectif : Élaborer et mettre en pratique des mesures de l'importance relative dérivées des méthodes ADD et MANOVA qui soient fondées sur les moyennes tronquées et les covariables de Winsor pour évaluer l'importance d'un domaine dans les données multivariées non normales.

Méthodologie : Des méthodes ADD et MANOVA non sensibles (c.-à-d. robustes) aux écarts par rapport à l'hypothèse de normalité multivariée ont été élaborées en remplaçant les estimations de la moyenne par les moindres carrés par des moyennes tronquées, et les covariables par des covariables de Winsor. Les mesures de l'importance des variables dérivées des coefficients de ces méthodes ADD et MANOVA robustes ont été utilisées pour classer par ordre d'importance les variables pour les données multivariées non normales. Les mesures de l'importance des variables fondées sur les moindres carrés et les estimateurs robustes ont été illustrées à l'aide des données de l'étude en cours Manitoba Inflammatory Bowel Disease Cohort, une étude de cohorte longitudinale visant à évaluer les facteurs psychosociaux prédictifs des problèmes de santé. Nous avons comparé les participants de cette étude ayant déclaré une maladie évolutive ( $\mathrm{n}=265)$ ou non évolutive ( $\mathrm{n}=116)$ en utilisant les quatre domaines du questionnaire sur les maladies inflammatoires de l'intestin (IBDQ, Inflammatory Bowel Disease Questionnaire) et les huit domaines du questionnaire SF-36 (Medical Outcomes Study 36-item Short Form), ce dernier évaluant les aspects physique et mental de la santé et du bien-être des participants.

Résultats : Lorsque nous avons utilisé les mesures de l'importance relative fondée sur les estimateurs des moindres carrés pour évaluer l'importance des domaines, les domaines des symptômes intestinaux dans l'IBDQ et les domaines de la santé en général dans le SF-36 ont été déterminés comme étant les domaines les plus importants. Ce sont les domaines émotionnels dans l'IBDQ et les domaines de la santé en général dans le SF-36 qui ont été déterminés comme étant les domaines les plus importants pour distinguer les deux groupes (maladie évolutive et maladie non évolutive) lorsque les mesures de l'importance des variables fondées sur les moyennes tronquées et les covariables de Winsor ont été utilisées. Le classement par ordre d'importance des domaines restants a varié selon les mesures de l'importance des variables et les méthodes d'estimation.

Conclusion : Ces mesures de l'importance relative peuvent être utilisées pour choisir un sous-groupe restreint de domaines qui distinguent le mieux les groupes d'après les données non normales sur la QdVLS. Une recherche additionnelle est nécessaire en vue d'étudier les propriétés de ces mesures dans différentes conditions d'analyse de données.

Mots-clés : biostatistique, population, santé publique

Rattachement des auteurs : 


\title{
Utilisation des soins de santé féminine par les femmes infectées par le VIH recevant un traitement antirétroviral en Colombie-Britannique
}

\author{
X. Wang, M.H.P. (13); K. A. Salters, M.H.P. (14); H. Wang, M. Sc. (14); W. Zhang, M. Sc. (14); N. Pick, M.D. (15, 16); J. S. \\ Montaner, M.D. (14, 15); R. S. Hogg, Ph. D. (13, 14); A. Kaida, Ph.D. (13)
}

*Ce résumé se trouve dans une version complète de l'article avec la citation suivante : Wang X, Salters KA, Zhang W, McCandless L, Money D, Pick N, Montaner JSG, Hogg RS, Kaida A. Women's Health Care Utilization among Harder-to-Reach HIV-Infected Women ever on Antiretroviral Therapy in British Columbia. AIDS Research and Treatment. 2012; doi:10.1155/2012/560361

Introduction : Les femmes représentent plus de $50 \%$ des personnes infectées par le $\mathrm{VIH}(\mathrm{VIH}+)$ à l'échelle mondiale et environ $22 \%$ à l'échelle nationale. L'existence d'expériences uniques à ces femmes VIH+ est bien établie : comparativement aux femmes non infectées par le VIH, les femmes VIH+ sont plus susceptibles de présenter des troubles gynécologiques et des problèmes liés à la ménopause, telle l'ostéoporose. Cependant, plusieurs études ont suggéré que les soins de santé féminine étaient sous-utilisés par les femmes VIH+ même s'il a été démontré que l'utilisation appropriée de ce type de soins réduisait le fardeau des maladies liées à l'infection par le VIH.

Objectif : Estimer la fréquence et les covariables de l'utilisation des soins de santé féminine par les femmes VIH+ ayant reçu un traitement antirétroviral (TAR) en Colombie-Britannique (C.-B.).

Méthodologie : L'étude longitudinale LISA (Longitudinal Investigations of Supportive and Ancillary Health Services) regroupe des personnes infectées par le VIH ayant reçu un TAR dans différentes cliniques en C.-B. Les données de l'enquête transversale sur les facteurs sociodémographiques, l'utilisation des services de soutien et la qualité de vie ont été couplées aux données cliniques longitudinales sur le VIH disponibles par l'intermédiaire du programme provincial Drug Treatment Program. Aux fins de cette analyse, l'inclusion des données a été limitée aux données des sujets de l'étude LISA identifiés comme étant de sexe féminin. Le paramètre évalué était l'utilisation actuelle des soins de santé féminine. Les réponses dichotomiques (oui ou non) étaient fondées sur les réponses à la question de l'enquête LISA : " J'ai un médecin que je vois régulièrement pour des soins de santé féminine ». Les covariables indépendantes incluaient des caractéristiques personnelles (âge, origine ethnique, autorité sanitaire, résidence en milieu rural ou urbain, état matrimonial), des variables sociodémographiques (scolarité, emploi, revenu, stabilité de résidence, sécurité alimentaire), des variables psychosociales (stigmatisation, perception de problèmes ou de cohésion avec le voisinage, qualité de vie), des variables relatives à l'abus de substances (alcool, drogue illicite, drogue injectable), des variables relatives à la santé sexuelle (activité sexuelle, utilisation de préservatifs, antécédents de commerce du sexe, intention de grossesse, nombre d'accouchements, antécédents d'infections transmissibles sexuellement, résultat anormal au test Pap dans les 6 derniers mois), des variables relatives à la santé mentale (symptômes de dépression) et des variables cliniques relatives au VIH (TAR, nombre de cellules CD4+, charge virale plasmatique, suppression virale, durée de l'immunodépression). Des analyses bidimensionnelles et des analyses de régression logistique multidimensionnelles ont été effectuées en vue de déterminer les facteurs associés à l'utilisation des soins de santé féminine.

Résultats : Parmi les 231 participantes, $77 \%$ recevaient régulièrement des soins de santé féminine. L'âge médian était de 41 ans, 49 \% étaient d'origine autochtone, $72 \%$ avaient un revenu annuel inférieur à 15000 \$, $62 \%$ avaient une résidence stable et $23 \%$ avaient une sécurité alimentaire. Dans le cadre de l'analyse multidimensionnelle, les facteurs associés à l'utilisation de soins de santé féminine englobaient les suivants : vivre à l'extérieur du territoire de l'autorité sanitaire de l'île de Vancouver (rapport de cotes $[\mathrm{RC}]=0,12$; intervalle de confiance [IC] à $95 \%$ : 0,04 à 0,37), ne pas consommer de drogues illicites (RC : 0,42; IC à $95 \%$ : $0,19$ à 0,92$)$, avoir un revenu annuel plus élevé (RC : 6,73; IC à $95 \%: 1,85$ à 24,54) et avoir une grande confiance en le fournisseur de soins de santé (échelle QdV) (RC : 1,03; IC à $95 \%$ : 1,00 à 0,05).

Conclusion : Malgré la fréquence relativement élevée de l'utilisation des soins de santé féminine par les femmes VIH+ recevant un TAR en C.-B., il existe toujours une lacune dans les services de soins de santé sur les plans géographique et social. Pour intégrer efficacement les soins de santé féminine dans le cadre des soins systématiques aux personnes VIH+,

Rattachement des auteurs :

13. Faculté des sciences de la santé, Université Simon Fraser, Burnaby (Colombie Britannique), Canada

14. Centre d'excellence sur le VIH/sida de la Colombie Britannique, Vancouver (Colombie Britannique), Canada

15. Faculté de médecine, Université de la Colombie Britannique, Vancouver (Colombie Britannique), Canada

16. Oak Tree Clinic, BC Women's Hospital and Health Centre, Vancouver (Colombie Britannique), Canada

Correspondance : Xueto Wang; courriel : wangxtk@gmail.com 
on doit adapter les programmes et les services aux besoins des femmes en répondant aux déterminants sociaux et structurels relatifs à leur santé.

Mots-clés : épidémiologie sociale et comportementale, santé des femmes, recherche sur les services de santé 